\title{
Beobachtungen auf der Sternwarte der k. u. k. Kriegs-Marine in Pola.
}

Die Beobachtungen nahmen am Abend des 13. November 1898 ihren Anfang. Diese Nacht war jedoch grösstentheils bewölkt und keine Sternschnuppe zu sehen.

Am 14. d. M. umzog sich der Himmel erst gegen I $^{\text {h }}$ und auch in dieser Nacht war kein Erfolg zu verzeichnen.

Erst am I 5 . November blieb die Nacht bis $18^{\mathrm{h}} \mathrm{m}$. P. Z. ziemlich klar; von $\mathrm{I}^{\mathrm{h}} \mathrm{m}$. P. Z. an bedeckten leichte Wolkenschieier den Himmel, die jedoch nicht störten. Um $18^{\mathrm{h}}$ m. P. Z. trat vollständige Bewölkung ein.

Die Aufschreibungen wurden tourenweise von je einem Beobachter durchgefuhrt, so dass wohl nur ein kleiner Theil der wirklich erfolgten Meteoritenfälle zur Wahrnehmung und Einzeichnung gelangte; das Hauptaugenmerk war dabei auf die nähere Umgebung des Sternbildes Leo major gerichtet.

Von $8^{\mathrm{h}}$ Abends bis ungefähr $\mathbf{I}^{\mathrm{h}} \mathrm{m}$. P. Z. kamen die Meteoriten aus verschiedenen Gegenden und erst von letzter
Zeit an (das Sternbild war ca. $\mathbf{2}^{\mathrm{h}} \mathrm{m}$. P. Z. aufgegangen) nahm der grösste Theil seinen Ursprung im Löwen; eine kleine Anzahl der von $I^{\mathrm{h}}$ bis $14^{\mathrm{h}} 30^{\mathrm{m}} \mathrm{m}$. P. Z. beobachteten Fälle kam aus der Gegend des Auriga. Die weiter bis $18^{\mathrm{h}}$ m. P. $Z$. verzeichneten Sternschnuppen waren fast lauter Leoniden und das Maximum der Fälle trat um ungefähr $16^{\mathrm{h}} 4^{8^{\mathrm{m}}}$ ein. Im Ganzen waren $8_{3}$ Meteoritenerscheinungen wahrgenommen worden, unter denen 34 der Gegend des Löwen entstammten. Nachstehend angefuhrte genauer verzeichnete 22 Bahnen bildeten das Material, aus dem der Radiant graphisch bestimmt wurde. Er ergab sich als in $\operatorname{der}$ Position $\alpha=10^{\mathrm{h}} \mathrm{I}^{\mathrm{m}}$; $d=+26^{\circ} 30^{\prime}$, also sehr nahe dem Stern $\mu$ Leonis liegend. Am 16. November blieb der Himmel die ganze Nacht vollständig bewölkt.

Beobachter waren die Herren Linienschiffs-Fähnriche Eugen Kavic, Karl Stockert und Felix Neuffer.

Verzeichniss der wahrgenommenen Leoniden 1898 Nov. 15

\begin{tabular}{|c|c|c|c|c|c|c|c|c|c|c|c|}
\hline \multirow{2}{*}{ Nr. } & \multirow{2}{*}{$\begin{array}{l}\text { Mittlere } \\
\text { Zeit Pola }\end{array}$} & \multicolumn{4}{|c|}{ Meteoritenbahn } & \multirow{2}{*}{ Nr. } & \multirow{2}{*}{$\begin{array}{c}\text { Mittlere } \\
\text { Zeit Pola }\end{array}$} & \multicolumn{4}{|c|}{ Meteoritenbahn } \\
\hline & & \multicolumn{2}{|c|}{ Anfangspunkt } & \multicolumn{2}{|c|}{ Endpunkt } & & & \multicolumn{2}{|c|}{ Anfangspunkt } & \multicolumn{2}{|c|}{ Endpunkt } \\
\hline $\mathbf{I}^{*}$ & $8^{h} 57^{m}$ & - & $\overline{6-0}$ & $-\overline{6}$ & $\overline{6}$ & $62^{*}$ & $14^{\mathrm{h}} 50^{\mathrm{m}}$ & - & - & - & - \\
\hline 24 & I 2 I I & 940 & +57.0 & 816 & +66.0 & $66^{*}$ & 1522 & 一 & - & - & - \\
\hline 25 & 122 I & I I I 2 & +42.0 & 1156 & +48.0 & $67 *$ & I 535 & - & - & - & - \\
\hline 26 & I $2 \quad 24$ & 1028 & +45.0 & 1052 & +52.5 & $68 *$ & 1540 & - & - & - & - \\
\hline $3^{6}$ & 138 & - & - & - & - & 72 & 1633 & 530 & -12.0 & 520 & -17.0 \\
\hline $42^{*}$ & 1326 & - & - & - & - & $73^{*}$ & 1635 & - & - & - & - \\
\hline 47 & 1344 & 851 & -3.0 & 842 & -9.0 & 74 & 1645 & I3 8 & +38.0 & I 328 & +40.5 \\
\hline 48 & 1353 & 935 & -3.0 & 924 & -12.0 & 76 & 1649 & $95^{2}$ & +35.0 & $93^{8}$ & +27.0 \\
\hline $5^{\circ}$ & 146 & $75^{2}$ & -18.5 & 740 & $-2 \mathrm{I} .0$ & 77 & I 653 & 1128 & +35.0 & 1142 & -39.0 \\
\hline $5 \mathrm{I}$ & 148 & 840 & 0.0 & 832 & -4.0 & 78 & 170 & I 33 & +49.0 & 1357 & +54.0 \\
\hline $56 *$ & 1432 & - & - & - & - & $82 *$ & 1735 & - & - & - & - \\
\hline $57^{*}$ & 1437 & - & - & - & - & $83^{*}$ & 1735 & - & - & - & - \\
\hline $6 I$ & I4 46 & II 20 & +32.0 & 1140 & $+3^{2} \cdot 5$ & & & & & & \\
\hline
\end{tabular}

Pola, 1898 November 18.

\section{Frhr. von Benko.}

*) Leoniden, deren Positionen nicht sicher genug festgestellt sind, theils wegen Verschwindens im Horizont, theils wegen ungenauer Einzeichnung, und die daher bei der Construction des Radianten ausgelassen wurden.

\section{Beobachtungen auf der Sternwarte in Taschkent.}

L'observation de cet essaim, commencée le I 3 novem. bre, m'a montré tout de suite que le nombre des Léonides était plus grand que celui des deux années précédentes.

D'après mes observations à peu près 15 Léonides sont tombés par heure.

Leur répartition par ordre de grandeur a été: 19 Léonides de ${ }^{r e}$ grandeur

\begin{tabular}{|c|c|c|c|}
\hline 5 & 》 & 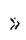 & $2^{\mathrm{e}}$ \\
\hline I I & » & 》 & $3^{\mathrm{m} \theta}$ \\
\hline 9 & 》 & 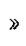 & $4^{\mathrm{me}}$ \\
\hline 2 & $\gg$ & » & $5^{\mathrm{me}}$ \\
\hline
\end{tabular}

Ies Léonides au-dessous de la $4^{\text {me }}$ grandeur sont difficiles à observer. Les météores n'appartenant pas à cet essaim n'ont pas été comptés.

Pour ce qui concerne la couleur des Léonides, tous les brillants étaient de la couleur un peu bleuâtre, à l'exception d'un seul dont la nuance était jaunâtre, mais qui ne faisait pas sûrement partie de cet essaim. Cette observation confirme bien celle de M. Hansky (C. R. CXXV, 759). Mon impression est que la couleur des Léonides se rapprothe beaucoup de celle de Sirius.

Quant à leur vitesse, elle a été très variable. 
